\title{
Eosinophilic annular erythema in childhood - Case report ${ }^{*}$
}

\author{
Alvaro Abarzúa ${ }^{1}$ \\ Sergio Silva ${ }^{1}$
}

\author{
Laura Giesen ${ }^{1}$ \\ Sergio González ${ }^{1}$
}

\begin{abstract}
Eosinophilic annular erythema is a rare, benign, recurrent disease, clinically characterized by persistent, annular, erythematous lesions, revealing histopathologically perivascular infiltrates with abundant eosinophils. This report describes an unusual case of eosinophilic annular erythema in a 3-year-old female, requiring sustained doses of hydroxychloroquine to be adequately controlled.
\end{abstract}

Keywords: Eosinophilia; Erythema; Pediatrics

\section{INTRODUCTION}

Eosinophilic annular erythema is a rare type of skin eosinophilic condition, characterized by recurrent or persistent urticarial annular lesions. Originally described in children, it is characterized by the presence of annular erythematous lesions, affecting predominantly trunk and proximal extremities. Lesion durations range from 4 to 12 months, and are associated with significant tissue eosinophilia. Only six cases of annular erythema with tissue eosinophilia have been published in children. The case of a 3-year-old female with eosinophilic annular erythema is herein described.

\section{CASE REPORT}

A 3-year-old female with no morbid history presented with red spots of approximately 1 month's evolution on both legs. Physical examination revealed erythematous annular papules and plaques with a pigmented and atrophic central area on both thighs. Laboratory tests showed normal blood counts without eosinophilia, ANA (-), ENA (-). Given the persistence and increasing size of the lesions, extending to the forearms and frontal and posterior surfaces of both thighs and legs, a skin biopsy was performed. Histopathological findings included dermal edema, interstitial and perivascular infiltrates of lymphocytes and eosinophils, and mast cell increase, all consistent with urticaria. An ad hoc diet was instated for one month, together with antihistamine and oral prednisone $(1 \mathrm{mg} / \mathrm{Kg})$. Initially, a good response to corticosteroids with a reduction of skin lesion sizes was observed, but a relapse shortly thereafter was recorded. Hence, hydroxychloroquine $50 \mathrm{mg} /$ day was initiated. The patient responded favorably, but presented a new outbreak (Figure 1). A second skin biopsy was performed, showing a dense, superficial and deep perivascular inflammatory infiltrate composed

Received on 15.09.2014

Approved by the Advisory Board and accepted for publication on 03.12.2014

* Work performed at the Departments of Dermatology and Pathology, Faculty of Medicine, Pontificia Universidad Católica de Chile - Santiago, Chile. Financial Support: None.

Conflict of Interest: None.

1 Pontificia Universidad Católica de Chile - Santiago, Chile.

C2016 by Anais Brasileiros de Dermatologia 
of lymphocytes and abundant eosinophils, without "flame figures" consistent with eosinophilic annular erythema (Figure 2). Currently, the patient is under maintenance doses of hydroxychloroquine (50mg/day) with a sustained, positive clinical response.

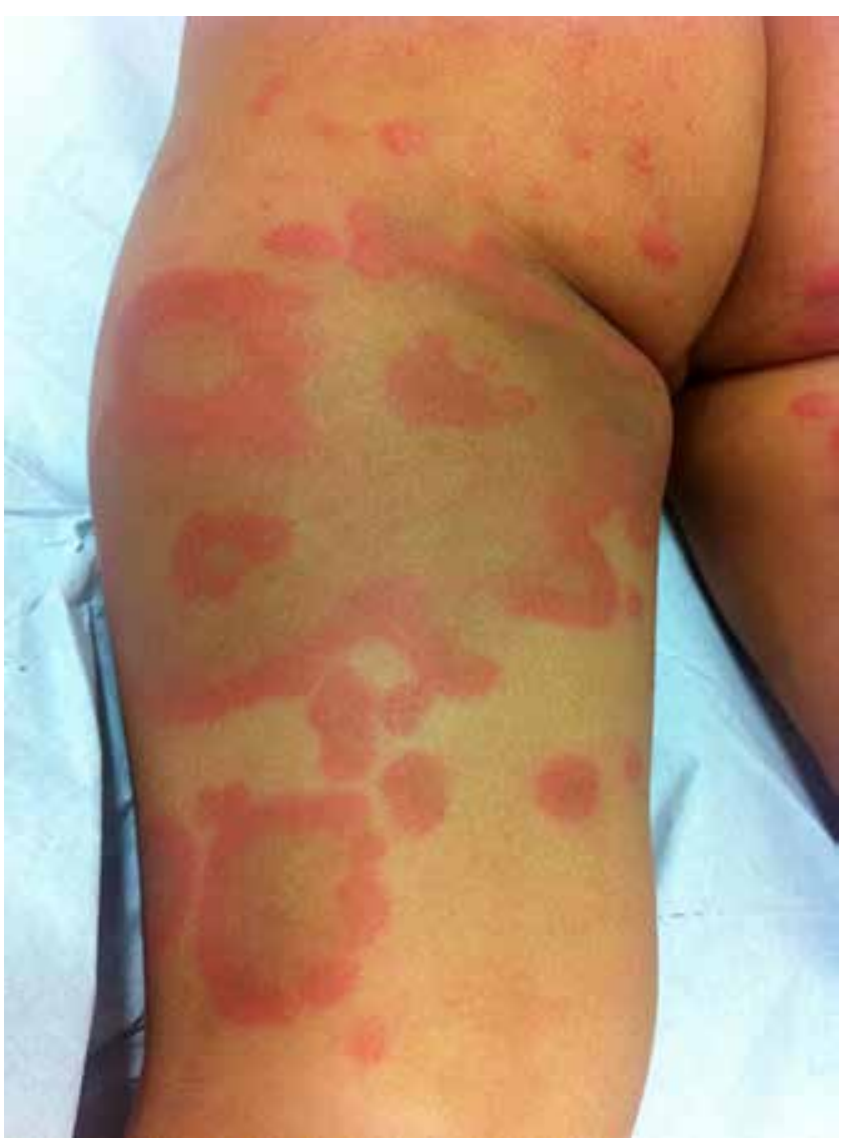

Figure 1: Few papules and extensive annular erythematous and infiltrated plaques with central hyperpigmentation on anterior and posterior aspects of both thighs and legs

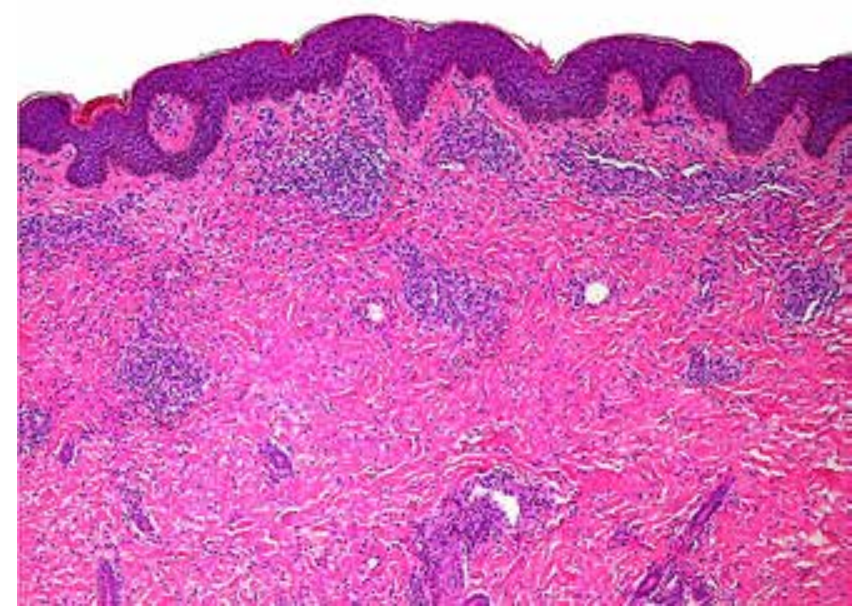

FIGURE 2: Skin biopsy showing normal epidermis and superficial and deep lymphocytic infiltrates with numerous eosinophils, HE 100x

\section{DISCUSSION}

Eosinophilic annular erythema is a rare, benign and recurrent disease. Originally described in children, but also seen in adults, it is characterized by the presence of annular erythematous lesions, affecting predominantly the trunk and proximal areas of the extremities. Lesion durations range from 4 to 12 months. An associated, significant tissue eosinophilia also appears. Only six cases of annular erythema with tissue eosinophilia have been published in children. The latest case involved a 4-year-old boy, described by Kunz et al. Since 2000, when the first case of eosinophilic annular erythema was reported in an adult woman, no further cases involving children have been described. ${ }^{1-4}$ Histopathology is quite characteristic, revealing a superficial, deep, perivascular, inflammatory infiltrate composed of lymphocytes and abundant eosinophils. "Flame figures" are unusual and were first described by Rongioletti et al. in two out of four adult patients, and later by El-Khalawany et al. in seven out of ten adult patients with well-developed, long-standing lesions. ${ }^{1-4}$ One important differential diagnosis is annular erythema in childhood, a rare entity that differs from eosinophilic annular erythema due to the presence of lesions that feature predominantly in children aged under 1, entailing spontaneous resolution and eosinophilic infiltration. ${ }^{3}$ The main differential histopathological diagnosis is Wells' syndrome, also known as eosinophilic cellulitis, where tissue eosinophilia and the presence of "flame figures" are characteristic. ${ }^{5}$ Wells' syndrome is uncommon in childhood and presents with infiltrated plaques resembling cellulitis, with or without blisters. It reveals a tendency toward spontaneous resolution within months. Wells' syndrome has been described in association with numerous conditions, including arthropod bites, drug allergy, neoplasia, parasites and HIV infection. ${ }^{6}$ Histopathologically, the inflammation extends into the hypodermis with a perivascular and/ or diffuse lymphocytic and eosinophilic infiltrate, entailing "flame figures", which are common but not specific to the syndrome. Wells' syndrome responds to systemic steroids. In the case described, no flame figures were observed and no identifiable cause could be determined. Insect bites should also be considered in the differential diagnosis. Clinical presentation includes papules, nodules, cellulitis-like plaques and lesions with blistering. Histopathologically, the inflammatory infiltrates may also be dense, with numerous eosinophils, as well as prominent papillary edema and subepidermal blisters. In our case, the lesions were large plaques and no indications of tick bites or similar arthropod accident were detected in the anamnesis. Currently, Wells' syndrome and eosinophilic annular erythema entities are regarded as different morphological expressions of a spectrum of eosinophilic dermatoses, thus reflecting the wide clinical and histopathological polymorphism, and the lack of a clear explanation for their pathogenesis. The distinction between eosinophilic annular erythema and annular erythema in childhood is arbitrary. ${ }^{5}$ Among the most accepted drugs to treat eosinophilic annular erythema are chloroquine and hydroxychloroquine, both capable of inhibiting the chemotaxis of eosinophils. ${ }^{3}$ Despite their demonstrated effectiveness, these drugs are often associated with an early recurrence of lesions after withdrawal. ${ }^{2,3,4} \square$ 


\section{REFERENCES}

1. Ríos-Martína J, Ferrándiz-Pulido L, Moreno-Ramírez D. Aproximación al diagnóstico dermatopatológico de las lesiones figuradas. Actas Dermosifiliogr. 2011;102:316-24.

2. El-Khalawany M, Al-Mutairi N, Sultan M, Shaaban D. Eosinophilic annular erythema is a peculiar subtype in the spectrum of Wells syndrome: a multicentre long-term follow-up study. J Eur Acad Dermatol Venereol. 2013;27:973-9.

3. Rongioletti F, Fausti V, Kempf W, Rebora A, Parodi A. Eosinophilic annular erythema: An expression of the clinical and pathological polymorphism of Wells syndrome. J Am Acad Dermatol. 2011;65:e135-7.

4. Toledo-Aberola F, Betlloch-Mas I. Eritemas anulares de la infancia. Actas Dermosifilogr. 2010;101:473-84.

5. Consigny $\mathrm{S}$, Courville $\mathrm{P}$, Young $\mathrm{P}$, Richard $\mathrm{C}$, Gauthier V, Maillard V, et al. Formes anatomo-cliniques du syndrome de Wells. Ann Dermatol Venereol. 2001;128:213-6.

6. Weedon D. Weedon's Skin Pathology. 3rd ed. London: Churchill Livingstone Elsevier, 2010.
MAILING ADDRESS:

Laura Giesen

Av. Vicuña Mackenna 4686.

San Joaquín

Santiago, Chile.

E-mail: giesen.laura@gmail.com

How to cite this article: Abarzúa A, Giesen L, Silva S, González S. Eosinophilic annular erythema in childhood Case report. An Bras Dermatol. 2016;91(4):503-5 